\title{
Inhalt
}

Anja Ballis, Nazli Hodaie

MehrSpracheN -1

Mark Häberlein

Fremdsprachenlernen in der Frühen Neuzeit: Bildungsverläufe, Lehrende und Lernende -9

Annemarie Saxalber

Was bedeutet sprachliche Grundbildung in einem mehrsprachigen

Bildungskontext? - 23

Tanja Angelovska, Claudia Maria Riehl

Zum Panel MehrSpracheN und Erwerbsprozesse: Dynamik, Individualität und Variation - 41

Nikolas Koch, Till Woerfel

Der Einfluss konstruktioneller Gebrauchsmuster in L1 und L2 auf die Verbalisierung intransitiver Bewegung bilingualer türkisch-deutscher Sprecher(innen) - 61

Anja Steinlen, Thorsten Piske

Deutsch- und Englischleistungen von Kindern mit und ohne Migrationshintergrund im bilingualen Unterricht und im Fremdsprachenunterricht: Ein Vergleich -85

Nazli Hodaie, Monika Raml

Von ,Sprachverfall' und Sprachwandel: Zum Panel MehrSpracheN als Varietäten des Deutschen — 99

Doris Grütz

Diglossie in der Deutschschweiz. Standardsprache versus Mundart - ein Problem in der Schule? —113

Ute Hofmann geil, krass oder porno, alder? Veränderungen kommunikativer Strategien und Handlungskompetenz -133 
Jürgen Joachimsthaler, Wendelin Sroka

Zum Panel MehrSpracheN im historischen Wandel —-151

Anna Maria Harbig

Kulturelle Wiedergeburt. Die mehrsprachigen Lehrbücher der griechischkatholischen Pfarrschulen in Galizien 1815-1848 — 165

\section{Blaise Extermann}

Handel, Technik und Mehrsprachigkeit. Fremdsprachenlernen in der Schweiz in der Zeit der zweiten industriellen Revolution 1880-1914 181

Monika Angela Budde

Zum Panel MehrSpracheN im Fach -197

Monika Angela Budde, Maike Busker

Fach-ProSa: Ein Modell zur fachbezogenen Professionalisierung zur

Sprachförderung in der Lehramtsausbildung der Fächer Chemie und

Deutsch 209

Christina Keimes, Volker Rexing

Textrezeptive Anforderungen in der Ausbildung. Eine Studie zur Bedeutung von

Lesekompetenz in gewerblich-technischen Ausbildungsberufen -227

Susanne Becker, Doris Fetscher

Zum Panel MehrSpracheN im Zeichen von Migration. Die Verhandlung von Migration und Mehrsprachigkeit im Diskursfeld Schule — 247

Séverine Behra, Rita Carol, Dominique Macaire

Wie weit ist der Weg von der superdiversity zur Anerkennung der frühen Mehrsprachigkeit im französischen Vorschulkontext? - 261

Edina Krompák, Luca Preite

Legitime und illegitime Sprachen in der Migrationsgesellschaft — 279

Kurzbiographien -297 